\title{
Perfil de isotipos de imunoglobulinas e subclasses de IgG na leishmaniose tegumentar americana
}

\author{
Immunoglobulin isotype and IgG subclass profiles \\ in american tegumentary leishmaniasis
}

\author{
Maria Aparecida de Souza ${ }^{1}$, Adriano Gomes da Silva1, Sandra Regina Afonso-Cardoso ${ }^{1}$, \\ Silvio Favoreto Junior ${ }^{2}$ e Marcelo Simão Ferreira ${ }^{3}$
}

\begin{abstract}
RESUM0
O presente trabalho avaliou o perfil de anticorpos em amostras de soro de 37 pacientes com diagnóstico clínico confirmado ou compatível com leishmaniose tegumentar americana atendidos no Hospital de Clínicas da Universidade Federal de Uberlândia, MG. Os perfis das classes de imunoglobulinas e subclasses de IgG foram analisados pelo teste ELISAindireto, utilizando-se antígeno solúvel de Leishmania (Leishmania) amazonensis. A avidez dos anticorpos foi determinada pelo tratamento com uréia a $6 \mathrm{M}$, após incubação dos soros com 0 antígeno. Observou-se que 97\%, 94,6\%, 57,5 e 21,5\% das amostras testadas apresentaram anticorpos anti-Leishmania das classes IgE, IgG, IgA e IgM, respectivamente e, os perfis das subclasses de IgG demonstraram, IgG1>IgG3>IgG2>IgG4. Os anticorpos IgE anti-Leishmania de alta avidez corresponderam a 44,4\%. Por outro lado, IgG e IgA anti-Leishmania foram em sua maioria (62,8 e 47,8\%, respectivamente), de média avidez. A variação do perfil de isotipos, bem como a avidez das imunoglobulinas refletiu a complexidade da resposta imune humoral contra a leishmaniose tegumentar americana.
\end{abstract}

Palavras-chaves: Leishmaniose tegumentar americana. Avidez. Imunoglobulinas. Subclasses de IgG.

\begin{abstract}
The present work investigated the serum antibody profiles in 37 patients with American tegumentary leishmaniasis, who were attended at Hospital de Clinicas - Universidade Federal de Uberlandia, MG, Brazil. The immunoglobulin class and IgG subclass profiles were analyzed by indirect ELSA using Leishmania (Leishmania) amazonensis soluble antigen. The antibody avidity was determined by 6 Murea treatment after incubation with immunoenzymatic conjugate. It was observed that $97 \%$ of the serum samples presented anti-Leishmania antibodies for IgE class, 94.6\% IgG, 57.5\% IgA and $21.5 \%$ IgMclass. For IgG subclasses the profiles were in the following order of frequency: IgG1 >IgG3>IgG2>IgG4. High avidity of anti-Leishmania IgE antibodies was found in $44.4 \%$ of the samples. On the other hand, moderate avidity of specific IgG and IgA was observed in $62.8 \%$ and $47.8 \%$ of samples, respectively. These results indicate a very complex antibody response profile against American tegumentary leishmaniasis.
\end{abstract}

Key-words: American tegumentary leishmaniasis. Avidity. Immunoglobulin. IgG subclasses.

Parasitas do gênero Leishmania são protozoários intracelulares obrigatórios que causam um complexo de enfermidades no homem e animais denominadas leishmanioses. Essas infecções atingem cerca de 12 milhões de pessoas em todo o mundo e são consideradas uma das dez principais doenças tropicais pela Organização Mundial da Saúde ${ }^{27}$. De acordo com este órgão, a forma tegumentar dessa parasitose é a apresentação clínica mais comum, acometendo cerca de $75 \%$ dos casos e constituindo um grave problema de saúde pública. Aimportância dessa moléstia não se deve apenas à sua alta incidência e ampla

\footnotetext{
1. Laboratório de Imunologia do Instituto de Ciências Biomédicas da Universidade Federal de Uberlândia, Uberlândia, MG. 2. Cardiovascular Research Institute of University of California at San Francisco, San Francisco, California, USA. 3. Serviço de Infectologia do Hospital de Clínicas da Universidade Federal de Uberlândia, Uberlândia, MG, Brasil.

Apoio Financeiro: CNPq e Sustainable Science Institute ( SSI), California, USA.

Endereço para correspondência: Prof. Marcelo Simão Ferreira. R. Goiás 480/500 Centro, 38400-064 Uberlândia MG, Brasil.

Telefax: 34 3236-3151

e-mail: mferreira@ nanet.com.br

Recebido para publicação em 26/02/2004

Aceito em 16/11/2004
} 
distribuição geográfica, mas também à possibilidade de produzir lesões destrutivas e desfigurantes, com importante repercussão no quadro psicossocial do paciente ${ }^{3}$.

As principais espécies responsáveis pela forma tegumentar dessa enfermidade no Brasil são Leishmania (Viannia) braziliensis, Leishmania (Leishmania) amazonensis e Leishmania (Viannia) guyanensis. Dentre as espécies menos incidentes encontram-se Leishmania (V.) naiffi, Leishmania (V.) shawii e Leishmania (V.) lainsoni ${ }^{11}$.

Inicialmente, em Minas Gerais, a leishmaniose tegumentar americana (LTA) ocorria sob forma esporádica ou epidêmica em locais de desmatamento realizado para a construção de rodovias e abertura de áreas agrícolas ${ }^{15}$. Atualmente, esse perfil tem-se modificado, com aumento da incidência dessa enfermidade em áreas peri-urbanas, graças principalmente ao desequilíbrio ambiental ${ }^{16}$.

Testes sorológicos têm sido empregados, com considerável importância, no diagnóstico e em inquéritos epidemiológicos dessas enfermidades. Além disso, a análise de anticorpos antiLeishmania permite avaliar o curso evolutivo da infecção, bem como fornecer dados sobre as características de sua resposta imune. Variados perfis e níveis de imunoglobulinas específicas a Leishmania têm sido detectados em pacientes com LTA, parecendo refletir não só a carga parasitária, mas também a espécie envolvida, 0 tempo de infecção e fatores intrínsecos do próprio hospedeiro ${ }^{8}$.

Como em outras doenças infecciosas e parasitárias, 0 monitoramento da resposta de anticorpos a antígenos envolvidos nos diferentes estágios da infecção pode fornecer informações valiosas que podem auxiliar na compreensão dos mecanismos de controle do sistema imune sobre os parasitas ${ }^{12}{ }^{1820}$. Em leishmaniose visceral ( IV), tem sido bem caracterizado o padrão de resposta do tipo TH2, evidenciado pela forte depressão da imunidade celular, baixa produção de citocinas proinflamatórias, como interferon-gama ( IFN- ${ }^{3}$ ) e interleucina-2 (IL-2) e, por outro lado, pelo aumento da produção das citocinas IL-4 e IL-5, ativação policlonal de células B e hipergamaglobulinemia ${ }^{69}$. Além disso, a detecção de anticorpos IgE específicos para Leishmania em LV tem sido correlacionada com a fase ativa da doença ${ }^{2}$. Em LTA, fica difícil de se estabelecer um padrão específico de resposta imune, pela complexidade da enfermidade. Em casos de leishmaniose cutânea (LC), foi observado um padrão misto TH1 e TH2 de resposta ${ }^{21}$ com a detecção de anticorpos IgE $\mathrm{E}^{25}$. Contudo, o papel de anticorpos específicos na imunidade anti-Leishmania ainda não está completamente esclarecido ${ }^{26}$.

Desse modo, a análise da presença de marcadores sorológicos aplicáveis ao diagnóstico é de grande importância no estudo dessas doenças assim como no desenvolvimento de estratégias de controle epidemiológico. 0 presente trabalho analisou os perfis das classes de imunoglobulinas (Ig) e subclasses de IgG em pacientes com quadro clínico confirmado ou compatível com LTA atendidos no Hospital de Clínicas da Universidade Federal de Uberlândia.

\section{MATERIAL E MÉTODOS}

Amostras de soro. Para a utilização das amostras de soro de pacientes, houve aprovação do comitê de ética da Universidade
Federal de Uberlândia, Uberlândia, MG, de acordo com 0 processo número 109/2002. Todas as amostras foram colhidas e manipuladas de acordo com as normas de biossegurança ${ }^{5}$.

As amostras de soro foram obtidas de 37 pacientes com diagnóstico clínico confirmado ou compatível com LTA, caracterizado pela presença de ulceras cutâneas simples ou múltiplas, atendidos no Hospital de Clínicas da Universidade Federal de Uberlândia. As amostras de soro controles (20) foram provenientes do Laboratório de Imunologia-UFU, obtidas de voluntários saudáveis, os quais não possuíam histórico clínico compatível de leishmaniose e foram sorologicamente não reagentes para doença de Chagas pelos testes ELISA e imunofluorescência indireta.

Preparação do antígeno de Leishmania. Formas promastigotas de Leishmania (Leishmania) amazonensis ( IFLA/BR/67/PH8) foram cultivadas em meio BHI (Brain Heart Infusion, Oxoid LTD, Basingstoke, Hampshire, UK) suplementado com 10\% de soro bovino fetal (Cultilab, Campinas, BR) , 1\% de antibiótico e 1\% de L-glutamina (Gibco BRL-Life Technologies, New York, USA) a 25ํ até atingirem a fase estacionária. Os parasitas $\left(10^{9}\right)$ foram colhidos, lavados por centrifugação em salina balanceada de Hanks (HBSS), ressuspensos em $1 \mathrm{ml}$ de salina tamponada com Tris (TBS a 0,02M, pH 7,2) contendo $1,6 \mathrm{mM}$ de fluoreto fenil-metil-sulfonil (PMSF) e mantidos em banho de gelo por 10 minutos. Em seguida, os parasitas foram submetidos a 3 ciclos de congelamento ( $\mathrm{N}_{2}$ líquido) / descongelamento (banho-maria, $3^{\circ} \mathrm{C}$ ) e rompidos por 3 ciclos de sonicação a 80 hertz. 0 lisado foi centrifugado a 20.000 x g por 1 hora a $4^{\circ} \mathrm{C}$. 0 sobrenadante foi colhido, filtrado em membrana de $0,22 \mathrm{~mm}$ e, a concentração protéica determinada (Lowry, 1951) e estocado a -20C até o momento do uso.

Ensaio imunoenzimático. ELISA indireto: placas de poliestireno ( Montegrotto Terme, Padova, Italy) , com 96 poços foram sensibilizadas com $10 \mathrm{mg} / \mathrm{ml}$ ( $50 \mathrm{ml} /$ poço) de antígeno em tampão carbonato/bicarbonato $0,06 \mathrm{M}, \mathrm{pH}$ 9,6. As placas foram incubadas por 18 horas a $4^{\circ} \mathrm{C}$ em câmara úmida e, em seguida lavadas com salina tamponada com fosfatos (PBS a 0,15M, pH 7,2) contendo 0,05\% de Tween-20 (PBS-T). As amostras de soro foram adicionadas ( $50 \mathrm{ml} /$ poço, em duplicata) na diluição 1/50 (menor diluição de detecção de anticorpos específicos anti-Leishmania determinada por titulação das amostras de soros) em PBS-T contendo 10\% de soro de cabra normal (PBS-T-SCN) . Após incubação por 45 minutos a $37^{\circ} \mathrm{C}$, as placas foram novamente lavadas e 0 conjugado imunoenzimático (anticorpos policlonais anti-IgG, ou -IgM, ou -IgA, ou -IgE humana produzidos em cabra ou monoclonais anti-subclasses de IgG humana produzidos em camundongos, ligados à peroxidase, Zymed Laboratories, DNC. San Francisco, CA) diluído em PBS-T-SCN foi adicionado (50ml/poço) e posteriormente incubadas por 45 minutos a $37^{\circ} \mathrm{C}$. Em seguida, as placas foram lavadas novamente, sendo adicionado o substrato enzimático (peridrol em tampão citrato de sódio/acido cítrico, pH 5, contendo orto-fenilenodiamina (OPD), 50ml/poço). Após 0 desenvolvimento da cor por 15 minutos, a reação foi interrompida com $\mathrm{H}_{2} \mathrm{SO}_{4} 2 \mathrm{~N}$ e a leitura efetuada em leitor de microplaca a $492 \mathrm{~nm}$. Os resultados foram expressos por índice ELISA ( IE) calculado pela fórmula EI $=(\mathrm{S}-\mathrm{B}) /(\mathrm{N}-\mathrm{B})$, onde 
Sé a média dos valores da densidade óptica em cada amostra, B corresponde à média dos valores da densidade óptica do branco e N, a média dos valores de densidade óptica dos controles negativos. Os valores de cortes das reações foram estabelecidos pela somatória das densidades ópticas das amostras negativas mais três desvios padrões, amostras acima desses valores foram consideradas reativas.

Ensaio imunoenzimático. ELISA avidez: placas de poliestireno (Montegrotto Terme, Italy) , com 96 poços, foram sensibilizadas com antígeno e incubadas com as amostras de soro, como descrito anteriormente. Previamente à adição do conjugado, as placas foram tratadas com uréia a 6M em PBS por cinco minutos. Em seguida, foram lavadas e o conjugado imunoenzimático (anti-isotipos) foi adicionado e a reação desenvolvida. Os resultados foram expressos em índice de avidez (IA), determinados pela razão entre os valores da densidade óptica das amostras tratadas com uréia ( $\mathrm{U}^{+}$) e a densidade óptica das amostras não tratadas (U) e expresso em porcentagem ([IA = $\left.\mathrm{U}^{+} / \mathrm{U} \times 100\right]$ ), sendo arbitrariamente definidos que: IA menor que $40 \%$ considerados de baixa avidez, entre 41 e 70\%, de média avidez e maior que 70\%, de alta avidez.

Análise estatística. Para comparação entre a mediana dos índices ELISA (IE), obtidos nas classes e subclasses de imunoglobulina, foi utilizado o teste não paramétrico de Mann-Whitney e teste t para comparação das proporções dos índices de avidez das classes de anticorpos. Valores de $\mathrm{p}<0,05$ foram considerados estatisticamente significantes.

\section{RESULTADOS}

Aspectos clínicos dos pacientes. Na Tabela 1 estão apresentados os principais dados demográficos e as formas clínicas dos pacientes, podendo-se observar que o tempo de evolução da doença variou entre 1 mês e 10 anos, com acometimento principalmente de pacientes do sexo masculino (83,8\%). Aforma cutânea foi a manifestação clínica predominante $(86,5 \%)$ dos casos.

Tabela 1- Dados demográficos, tempo de evolução e formas clínicas dos pacientes com LTA.

\begin{tabular}{|c|c|c|c|c|c|c|}
\hline \multirow{2}{*}{$\begin{array}{l}\text { Número de } \\
\text { pacientes }\end{array}$} & \multicolumn{2}{|c|}{ Sexo } & \multirow{2}{*}{$\begin{array}{l}\text { Idade } \\
\text { (anos) }\end{array}$} & \multirow{2}{*}{$\begin{array}{l}\text { Tempo de } \\
\text { evolução }\end{array}$} & \multicolumn{2}{|c|}{ Forma clínica } \\
\hline & $\mathrm{M}$ & $\mathrm{F}$ & & & LC & LCM \\
\hline 37 & 31 & 6 & 25 a 70 & 1 mês a 10 anos & 32 & 5 \\
\hline
\end{tabular}

$\mathrm{LC}=$ leishmaniose cutânea; LCM = leishmaniose cutâneo-mucosa;

$\mathrm{M}=$ masculino; $\mathrm{F}=$ feminino.

Análise dos isotipos de imunoglobulinas. Foram analisadas 37 amostras de soro de pacientes pelo teste ELISA, os resultados estão expressos em Índice ELSA (IE) com seus respectivos pontos máximos e mínimos e, porcentagens de amostras reativas em cada classe de imunoglobulina (Figura 1). Das 37 amostras de soro analisadas foram detectados anticorpos das classes IgE e IgG anti-Leishmania em 97\% e 94,6\% respectivamente ( Figura 1, p<0,05) cujos valores de cortes para cada classe de imunoglobulina foram 0,036 para IgE e 0,054 para IgG. Em 21,5\% das amostras de soro dos pacientes foram

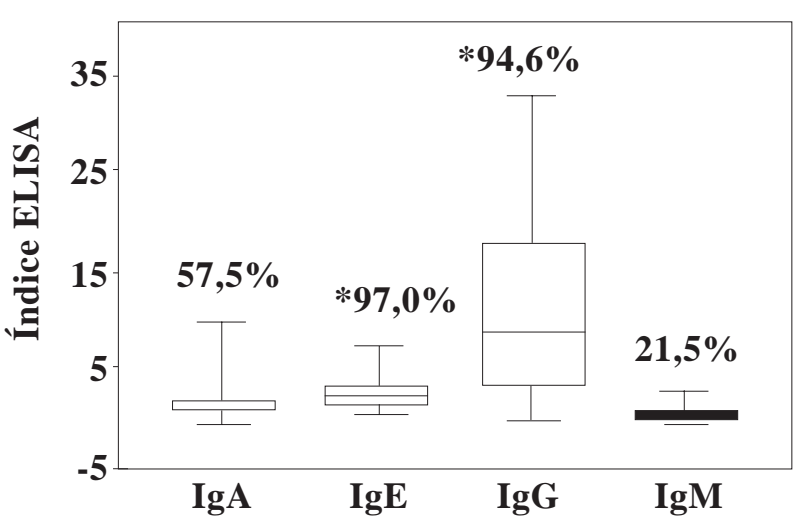

Figura 1 - Perfil das classes de anticorpos em pacientes com LTA. Os valores das medianas dos IE estatisticamente significantes estão assinalados por * quando $\mathrm{p}<0,05$, pelo teste de Mann-Whitney.

detectados anticorpos da classe IgM (valor de corte 0,143) e $57,5 \%$ (valor de corte 0,038 ) da classe IgA (Figura 1). Os maiores níveis de reatividade foram observados para a classe IgG quando as medianas dos índices ELISA (IE) foram comparadas às medianas das demais classes de anticorpos (Figura 1, p<0,05). A IgE anti-Leishmania, apresentou baixa reatividade para a maioria das amostras de soro (Figura 1). No entanto, ao se analisar 0 índice de avidez dessa imunoglobulina, observou-se que em sua maioria (44,4\%, $\mathrm{p}<0,05)$ apresentaram-se de alta avidez (Tabela 2). Por outro lado, IgG e IgA anti-Leishmania foram predominantemente de média avidez (62,8 e 47,8\%, respectivamente, Tabela 2; $p<0,05$ ).

Tabela 2- Perfil de avidez dos anticorpos anti-Leishmania observados em 37 pacientes portadores de LTA.

\begin{tabular}{lccc}
\hline & \multicolumn{3}{c}{ Avidez } \\
\cline { 2 - 4 } Isotipos & $<40 \%$ (baixa) & $41-70 \%$ (média) & $>70 \%$ (alta) \\
\hline IgA & $26,1 \%$ & $47,8 \%{ }^{*}$ & $26,1 \%$ \\
IgE & $19,4 \%$ & $36,1 \%$ & $44,4 \%^{*}$ \\
IgG & $17,1 \%$ & $62,8 \%{ }^{*}$ & $20,0 \%$ \\
\hline
\end{tabular}

${ }^{*} \mathrm{p}<0,05$

Subclasses de IgG. Foram analisadas 37 amostras de soro de pacientes pelo teste ELSA, os resultados estão expressos em Índice ELISA (IE), com suas respectivas porcentagens de amostras reativas em cada subclasse de IgG (Figura 2). A análise

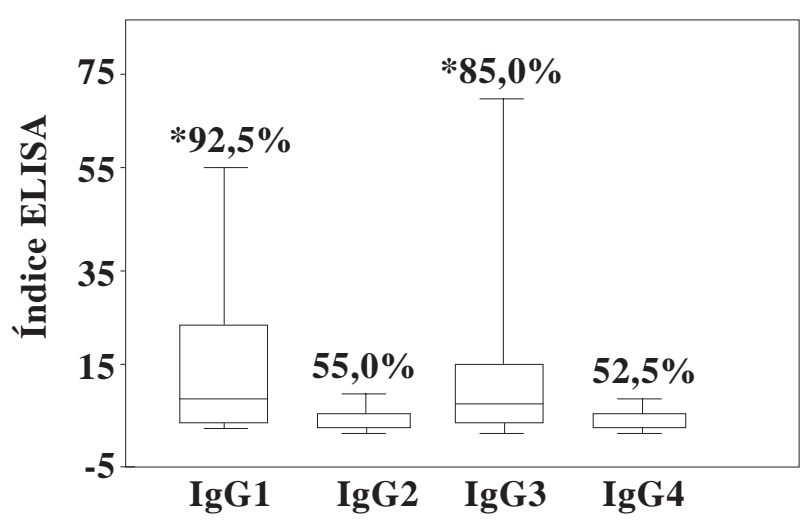

Figura 2 - Perfil das subclasses de IgG em amostras de soros de pacientes com LTA. Os valores das medianas dos IE estatisticamente significantes estão assinalados por * quando $\mathrm{p}<0,05$, pelo teste de Mann-Whitney. 
dos perfis de subclasses de IgG das 37 amostras de soro, demonstrou o predomínio de IgG1 em 92,5\% dos pacientes, seguido pela IgG3 com 85\% (Figura 2, p<0,05). As subclasses que apresentaram menores níveis de detecção foram IgG2 (55\%) e IgG4 ( $52,5 \%$ ). Desse modo, o perfil de isotipos observado foi IgG1>IgG3>IgG2>IgG4 (Figura 2). 0 índice de reatividade entre as subclasses foi significantemente maior $(p>0,05)$ para IgG1 e IgG3 quando comparados aos índices de detecção de IgG2 e IgG4, como demonstrado pelas medianas dos índices ELSA( IE) de cada subclasse de IgG ( Figura 2).

\section{DISCUSSÃ0}

A infecção por Leishmania normalmente, leva à indução de resposta imune complexa, caracterizada tanto por reações mediadas por células como pela produção de anticorpos. A natureza da resposta imune celular desempenha um papel fundamental na determinação da resposta imune humoral pela indução da produção de anticorpos de isotipos específicos de imunoglobulinas ${ }^{23}$. No presente estudo, foi avaliado 0 perfil dos isotipos das imunoglobulinas em 37 amostras de soro, no qual detectou-se que a classe IgE anti-Leishmania estava presente na maioria das amostras de soro de pacientes com LTA. Dados de literatura demonstram que existe uma correlação positiva entre altos índices de IgE e reação de Montenegro, sugerindo que estes anticorpos participam do processo de imunorregulação na leishmaniose cutânea ${ }^{25}$. No entanto, neste estudo não foi realizada esta prova nos pacientes, dificultando deste modo, estabelecer-se relação semelhante. Além disso, em pacientes nos estágios iniciais de LC, tem sido demonstrado maior produção de IL-10 em detrimento de IFN- $\gamma$, ocorrendo troca deste perfil ao longo da evolução da doença, onde citocinas proinflamatórias passam a serem predominantes ${ }^{22}$. Sendo assim, os achados de IgE antiLeishmania no presente estudo, podem estar associados aos processos iniciais da evolução da doença e consequentemente, ao perfil TH2 de resposta imune nesses pacientes. Por outro lado, em leishmaniose mucosa há uma mistura de perfil de resposta TH1 e TH2, antes e após tratamento ${ }^{1}$.

No estudo em apreço, em todos os pacientes com quadro clínico de leishmaniose mucosa foram detectados altos índices de IgA (57,5\%). Esses dados estão de acordo com outros autores ${ }^{19}$, que também demonstraram elevação dos níveis de anticorpos IgA-Leishmania específicos em pacientes com comprometimento mucoso.

Embora muitos dos pacientes apresentassem pouco tempo de evolução das lesões, altos níveis de anticorpos da classe IgM não foram detectados. Esses dados diferem de outros da literatura em que os níveis de IgM se correlacionam com a fase inicial da infecção ${ }^{10}$. No entanto, este fato pode ser explicado tanto pelas limitações do teste sorológico (ELISA), como pela preparação antigênica empregada nesse estudo.

Em LTA, a forte imunidade mediada por células e a predominância de isotipos IgG1, IgG2 e IgG3 nas leishmanioses cutâneas e mucocutâneas tem sido associadas ao perfil TH1 de resposta ${ }^{34}$. Já, 0 isotipo IgG4 relaciona-se às manifestações da leishmaniose cutânea difusa, associado ao perfil TH2 da resposta imune ${ }^{2325}$. Neste trabalho, as subclasses de IgG predominantemente detectadas também foram IgG1 e IgG3, em acordo com outros trabalhos ${ }^{23} 26$, que também demonstraram a predominância de anticorpos dessas subclasses na LC.

Em doenças infecciosas, tais como toxoplasmose e doença de Chagas, a avidez de anticorpos determina a fase da infecção, sendo considerada importante marcador sorológico ${ }^{13} 1417$. Em leishmaniose, a associação entre 0 quadro clínico do paciente com o perfil de imunoglobulinas, bem como, com sua avidez, é muito complexo. No presente estudo, não foi possível estabelecer correlação entre o tempo de evolução da lesão dos pacientes e a avidez das imuno globulinas, pois, nos casos de lesões recentes, entre 1 a 3 meses, foram observados anticorpos com altos índices de avidez. Além disso, em pacientes com até 10 anos de infecção foram detectados anticorpos de baixa avidez. Esses dados evidenciam a complexidade da resposta imune humoral em pacientes com LTA, mostrando que durante esse processo infeccioso, não há correlação entre maturação e mensuração de anticorpos, em termos de avidez, com a evolução da parasitose.

\section{REFERÊNCIAS BIBLIOGRÁFICAS}

1. Amato VS, Andrade Jr HF, Duarte MIS. Mucosal leishmaniasis: in situ characterization of the host inflammatory response, before and after treatment. Acta Tropica 85: 39-49, 2003.

2. Atta AMJr, D'Oliveira A, Correa J, Atta MLB, Almeida RP, Carvalho EM. Antileishmanial IgE antibodies: a marker of active disease in visceral leishmaniasis. The American Journal of Tropical Medicine and Hygiene 59: 426-430, 1998 .

3. Carvalho EM, Johnson WD, Barreto E, Marsden PD, Costa JL, Reed S, Rocha H. Cell-mediated immunity in American Cutaneous and mucosal leishmaniasis. Journal of Immunology 135:4144-4148, 1985.

4. Castes M, Agnelli A, Verde 0, Rondon AJ. Characterization of the cellular immune response in American cutaneous leishmaniasis. Clinical Immunology and Immunopathology 27:176-186, 1983.

5. Costa MAF. Qualidade em biossegurança.Qualitymark, Rio de Janeiro, 2000.

6. Galvão-Castro B, Sa Ferreira JA, Marzochi KF, Marzochi MC, Coutinho SG, Lambert PH. Polyclonal B cell activation, circulating immune complexes and autoimmunity in human American visceral leishmaniasis. Journal Clinical Experimental Immunology 56: 58066, 1984.

7. Gontijo B, Carvalho MLR. Leishmaniose tegumentar americana. Revista da Sociedade Brasileira de Medicina Tropical 36: 71-80, 2003.

8. Gutierrez Y, Salinas GH, Palma G, Valderrama LB, Santrich CV, Saraiva NG. Correlation between histopathology, immune response, clinical presentation, and evolution in Leishmania braziliensis infection. The American Journal of Tropical Medicine and Hygiene 45: 281-289, 1991.

9. Holaday BJ, Pompem ML, Geronimo S, Teixeira MJ, Souza AQ, Vasconcelos AW, Pearson RD, Abrams JS, Locksley RM. Potential role for interleukin10 in the immunosuppression associated with kalar-azar. Journal Clinical Investigation 92: 2626-2632, 1993.

10. Labrada M, Weigle K, Valderrama L, Saravia NG. Evaluation of immunoglobulin isotype specific to Leishmania in tegumentary American leishmaniasis. Memórias do Instituto Oswaldo Cruz 84: 409-416, 1989.

11. Lainson R, Shaw JJ. Evolution, classification and geographical distribution. In: Peters W, Killick-Kendrick R (eds) the Leishmaniasis in Biology and Medicine, Academic Press, London, p. 1-120, 1987. 
12. Lutz E, Ward, KNGrayJJ. Maturation of antibody avidity after primary human cytomegalovirus infection is delayed in immunosuppressed solid organ transplant patients. Journal Medical Virology 44:317-322, 1994.

13. Marcipar IS, Risso MG, Silber AM, Revelli S, Marcipar A, Antibody maturation in Trypanosoma cruzi-infected rats. Clinical and Diagnostic Laboratory Immunology 8: 802-805, 2001

14. Marcolino PT, Silva DAO, Leser PG, Camargo ME, Mineo JR. Molecular markers in acute and chronic phases of human toxoplasmosis: determination of immunoglobulin $\mathrm{G}$ avidity by western blotting. Clinical and Diagnostic Laboratory Immunology 7: 384-389, 2000.

15. Mayrink W, Williams P, Coelho MV, Dias M, Martins AV, Magalhães PA, Da CC, Falcão AR, Melo MN, Falcão AL. Epidemiology of dermal leishmaniasis in the Rio Doce Valley, state of Minas Gerais, Brazil. Annual Tropical Medical Parasitology 73: 123-137, 1979.

16. Maywald PG, Machado MI, Costa CJ, Gonçalves PM. Canine cutaneous and visceral leishmaniasis and Chagas disease from countries in the Triângulo Mineiro and Alto Paranaiba regions, Minas Gerais State, Brazil. Cadernos de Saúde Pública 12: 321-328, 1996.

17. Mechain B, Garin YJF, Robert-Gangneux F, Dupouy-Camet J, Derouin F. Lack of utility of specific immunoglobulin $\mathrm{G}$ antibody avidity for serodiagnosis of reactivated toxoplasmosis in immunocompromised patients. Clinical and Diagnostic Laboratory Immunology 7: 703-705, 2000.

18. Motran CC, Cerbán, FM, Rivarola H, Vottero de Cima E. Characterization of autoantibodies generated in mice by immunization with the $\mathrm{C}$-terminal region of Trypanosoma cruzi ribosomal P1 and P2 proteins. Clinical Immunology 91: 17-24, 1999.

19. O'Neil CE, Labrada M, Saraiva NG. Leishmania (Viannia) panamensisspecific IgE and IgA antibodies relation to expression of human tegumentary leishmaniasis. The American Journal of Tropical Medicine and Hvgiene 49: 181-188, 1993.

20. Paul M. Immunoglobulin $G$ avidity in diagnosis of toxoplasmic lymphadenopathy and ocular toxoplasmosis. Clinical and Diagnostic Laboratory Immunology 6: 514-518, 1999.

21. Pirmez C, Yamamura M, Uyemura K, Paes-Oliveira M, Conceição-Silva F, Modlin RL. Cytokine patterns in the pathogenesis of human leishmaniasis. Journal Clinical Investigation 91: 1390-1395, 1993.

22. Rocha PN, Almeida RP, Bacellar 0, de Jesus AR, Filho CD, Filho AC, Barral A, Coffman RL, Carvalho EM. Down-regulation of Th1 type of response in early human american cutaneous leishmaniasis. The Journal of Infectious Diseases 180: 1731-1734, 1999.

23. Rodriguez V, Centeno M, Ulrich M. The IgG isotypes of specific antibodies in patients with American cutaneous leishmaniasis: relationship to the cellmediated immune response. Parasite Immunology 18: 341-345, 1996.

24. Skeiky YAW, Benson DR, Costa JLM, Badaró R, Reed SG. Association of Leishmania heat shock protein 83 antigen and immunoglobulin G4 antibody titers in Brazilian patients with diffuse cutaneous leishmaniasis. Infection and Immunity 65: 5368-5370, 1997.

25. Sousa-Atta MLB, Salomé GS, D'Oliveira Jr A, Almeida RP, Atta AM, Carvalho EM Immunoglobulin Eantileishmanial antibody response in cutaneous leishmaniasis. Clinical and Diagnostic Laboratory Immunology 9: 101-104, 2002.

26. Trujillo C, Ramirez R, Velez ID, Berberich C. The humoral immune response to the kinetoplatid membrane protein-11 in patients with American Leishmaniasis and Chagas disease: prevalence of IgG subclasses and mapping of epitopes. Immunology Letters 70: 203-209, 1999.

27. World Health Organization. Leishmania and HIV in gridlock. UNAIDS, Geneva, Switzerland, 1998. 